\title{
Evaluation of co-morbidity indices in patients admitted for Chronic Obstructive Pulmonary Disease
}

\author{
R.G. Pinckney ${ }^{1}$, R. O’Brien ${ }^{1}$, J.F. Piccirillo2, B. Littenberg1
}

ABSTRACT: Evaluation of co-morbidity indices in patients admitted for Chronic Obstructive Pulmonary Disease. R.G. Pinckney, R. O'Brien, J.F. Piccirillo, B. Littenberg.

Background. There is limited and conflicting information on the use of co-morbidity instruments to predict mortality in patients with chronic obstructive pulmonary disease (COPD)

Methods. We sought to test the validity of the Charlson Index and another co-morbidity instrument, the Adult co-morbidity evaluation 27 (ACE-27), in patients admitted with COPD exacerbations. Co-morbidity scores were obtained by chart review. Information on mortality was re- trieved from the Social Security Death Index. We examined the predictive validity of the Charlson and the ACE27 using survival analysis.

Results. There were 112 patients eligible for the study. The ACE-27 but not the Charlson predicted survival, after adjusting for age, gender, and smoking history in Cox regression, hazard ratio $(95 \% \mathrm{CI})$ of 1.99 (1.17-3.39).

Conclusions. This study confirms earlier findings that the Charlson Index is not a reliable predictor of mortality in patients with COPD. However, the ACE-27 appears to be useful for predicting survival in this study.

Monaldi Arch Chest Dis 2004; 61: 4, 209-212.

Keywords: Pulmonary disease, chronic obstructive; co-morbidity; risk adjustment; prognosis.

1 General Internal Medicine, University of Vermont

2 Otolaryngology, Head and Neck Surgery, Washington University, USA.

Correspondence: Richard G. Pinckney MD, MPH; General Internal Medicine; University of Vermont; 371 Pearl St; Burlington, Vt 05401; USA; e-mail: richard.pinckney@vtmednet.org

\section{Introduction}

Chronic obstructive pulmonary disease (COPD) is a condition with significant mortality; yet, the majority of patients with COPD die from other diseases [1-4]. This evidence has lead to the further study of co-morbidity and COPD. Seventy three percent of patients with COPD have at least one co-morbid condition [5]. In COPD cohort studies, patients with increased co-morbidity have reduced survival [6-9]. Given the potential importance of co-morbidity when studying COPD, methods are needed to quantify the burden of co-morbidity for analysis.

There have been several approaches to quantifying the burden of co-morbidity in studies of obstructive lung disease. One method is to simply sum the number of co-morbid conditions. This has not been shown to predict mortality in patient with COPD $[10,11]$. Another approach has been to use a validated co-morbidty instrument. The Charlson Co-morbidity Index (CCI) is the only co-morbidity instrument previously used to study the relationship between co-morbidity and mortality in patients with COPD. Though most studies have shown an decrease in survival as the Charlson Co-morbidity Index rises [6-9] there have been some studies that have shown no association $[12,13]$. Though the CCI has been validated in many other populations $[14,15]$ it has limited content validity [16] which could explain its unreliable performance in patients with COPD. Many conditions have been shown to affect long term mortality; yet, the CCI captures only 16 different medical conditions. Furthermore, the severity of co-morbidities may affect long term survival. For example, a patient with severe heart failure awaiting transplant has a different prognosis than someone with asymptomatic heart failure [17]. The CCI only measures the severity of 2 of the 16 conditions it captures. An instrument which overcomes the limitations of the CCI may have a more consistent performance.

The Adult Co-morbidity Evaluation (ACE-27) [18] is a modified Kaplan Feinstein Index (KFI) [19]. It overcomes the limitations of the CCI by collecting the presence and severity of 27 different co-morbid conditions. We sought to examine whether the ACE-27 was predictive of survival in patients with COPD exacerbations. We also reexamined the predictive validity of the CCI.

\section{Methods}

We identified a retrospective cohort of patients admitted for COPD exacerbation in Burlington Vermont from January 1995 to June 1996. The subjects came from two hospitals in the City of Burlington, Vermont in the United States of America. Subjects were identified by searching the hos- 
pital administrative databases for principal diagnoses of bronchitis, emphysema, or respiratory failure using the International Classification of Diseases Ninth Version (ICD-9) codes 490, 491, 492, 496, $518.81-.84,786.09$, and 799.1. Identified admissions were included in the study if the diagnosis on the discharge summary was COPD exacerbation. In addition, the patient had to meet at least two of the following criteria adapted from the American Thoracic Society: increased frequency of cough, increased sputum production, increased dyspnoea, or respiratory failure [20]. Subjects had to be at least 35 years of age, have a previous smoking history, and no chest x-ray evidence of pulmonary edema, pneumonia, or bronchiectasis on admission. Subjects with a history of asthma were excluded as they have been shown to have a different prognosis [21-23] and their numbers were too small for appropriate subgroup analysis. Subjects were excluded if they had no social security number as this was needed to measure the outcome. Patients transferred from another hospital were excluded as the past records were often unavailable.

Information on co-morbidity and covariates were collected using standardized methods and forms. Cigarette use, sex, and age were obtained from the subjects' charts. Information on co-morbidity was collected from the admission note, prior admission records, and the problem list. Data for an APACHE II score was collected for a subgroup of these patients and reported elsewhere [24]. The Social Security Death Index (SSDI) was used to determine vital status and time of observation for survival analysis [25].

The Charlson co-morbidity score was calculated and analyzed in six categories as previously described $(0,1,2,3,4, \geq 5)[13]$. The ACE-27 co-morbidity score was calculated using the same methodology for the Kaplan-Feinstein Index [26]. The co-morbidity score is analyzed as a score of 0 3. Alternatively, the co-morbidity score can be dichotomized, those having a score of 3 have "prognostic co-morbidity." COPD was not considered a co-morbid condition for calculating the Charlson or ACE-27 co-morbidity scores.

\section{Analysis}

Survival analyses were performed. The time of observation for patients who died was the date of the admission to the date of death in the Social Security Death Index (SSDI). The time of observation for those who had no SSDI death record was the time of admission to the time of the SSDI search. The risk of death associated with co-morbidity, current smoking, total pack-years of smoking, age, and gender were each determined by univariate Cox regression. Covariates associated with $\mathrm{P}<.2$ were considered potential confounders of the relationship between co-morbidity and survival, and they were included in multivariate models with the co-morbidity scores. The proportional hazards assumption of the final models were tested using Schoenfeld residuals [27]. All analyses were performed using STATA 7.0 [28].

\section{Results}

There were 277 admissions that met ICD-9 search criteria. All charts were obtained and 165 patients were excluded for the following reasons: 101 did not meet clinical criteria for COPD exacerbation, 8 did not have a prior smoking history, 12 had a history of asthma, 24 had x-ray evidence of another diagnosis explaining symptoms, 19 had been transferred from other hospitals, and one did not have a social security number. The characteristics on the day of admission, hospital course, and mortality of the 112 eligible patients were summarized (table 1). The median Charlson score was 1, median ACE-27 was 2, and $29 \%$ of subjects had prognostic co-morbidity as determined by the ACE-27. At the end of the observation period, $41 \%$ of the subjects were alive with a mean observation time of 2.49 years.

Table 2 presents the associated frequencies of the Charlson and ACE-27 scores. We discovered that there is a large drop in survival from a Charlson score of 0 to 1 ( $58 \%$ to $21 \%$ respectively) with very little change in survival as the score increased beyond 1 . We also confirmed the similar survival among ACE-27 scores 0-2, but that survival dropped precipitously with a score of 3 . On this table, there is a suggestion that the ACE-27 may have an advantage over the CCI. There were 20 patients with ACE-27 prognostic co-morbidity that had low CCI scores (of 0-2) and these patients had a very low survival of $10 \%$ ). There was not a similar group of patients with high Charlson scores and low ACE-27 scores.

In univariate Cox regression, the $\mathrm{CCI}$ and ACE-27 were both predictive of mortality (table 3 ). However after adjusting for age, gender, and packyears of smoking in Cox proportional hazards models, only the ACE-27 was a statistically significant predictor of survival (table 4). Having prognostic co-morbidity increased the hazard of death to nearly double the risk of those not having this degree of co-morbidity. The Schoenfeld residuals

Table 1. - Characteristics of study population, $\mathrm{N}=112$

\begin{tabular}{ll}
\hline Characteristic & \multicolumn{1}{c}{ Result } \\
\hline Gender, \% female & $52 \%$ \\
Age in years, mean +/- SD & $69.4+/-8.48$ \\
Current smokers & $38 \%$ \\
Pack/Years, mean +/- SD & $68.4+/-33.5$ \\
Admitted to ICU & $18 \%$ \\
Intubated & $8 \%$ \\
Length of Stay in days, mean +/- SD & $10.9+/-29.8$ \\
Alive at discharge & $96 \%$ \\
Time to follow-up, mean +/- SD & 2.49 years $+/-1.6$ \\
Alive at follow-up & $41 \%$ \\
Charlson: median, range & $1,0-7$ \\
Charlson co-morbidity Present & $57 \%$ \\
ACE-27: median, range & $2,0-3$ \\
ACE-27: Prognostic co-morbidity & $29 \%$ \\
\hline
\end{tabular}


Table 2. - Frequency of Charlson and ACE-27 scores with percent survival at the end of the follow-up period

\begin{tabular}{|c|c|c|c|c|c|}
\hline \multirow[b]{2}{*}{ Charlson Score } & \multicolumn{5}{|c|}{ ACE-27 Score } \\
\hline & 0 & 1 & 2 & 3 & All \\
\hline 0 & $22(55 \%)$ & $16(63 \%)$ & $8(75 \%)$ & $2(0 \%)$ & $48(58 \%)$ \\
\hline 1 & & $11(18 \%)$ & $10(30 \%)$ & $7(14 \%)$ & $28(21 \%)$ \\
\hline 2 & & $4(75 \%)$ & $7(57 \%)$ & $11(9 \%)$ & $22(36 \%)$ \\
\hline 3 & & & $1(0 \%)$ & $5(40 \%)$ & $6(33 \%)$ \\
\hline 4 & & & & $5(40 \%)$ & $5(40 \%)$ \\
\hline$\geq 5$ & & & & $3(0 \%)$ & $3(0 \%)$ \\
\hline All & $22(55 \%)$ & $31(48 \%)$ & $26(50 \%)$ & $33(18 \%)$ & $112(41 \%)$ \\
\hline
\end{tabular}

Table 3. - Co-morbidity scores and risk of death, unadjusted

\begin{tabular}{lccc}
\hline Predictor & HR & $(95 \% \mathrm{CI})$ & $\mathrm{p}$ \\
\hline ACE-27 Prognostic co-morbidity & 2.18 & $(1.33-3.56)$ & 0.002 \\
ACE-27 co-morbidity Grade (0-3) & 1.35 & $(1.07-1.70)$ & 0.01 \\
Charlson co-morbidity Index $(0-\geq 5)$ & 1.18 & $(1.00-1.41)$ & 0.042 \\
\hline
\end{tabular}

Table 4. - Co-morbidity scores and adjusted risk of death

Risk of death determined using Cox proportional hazards models adjusting for age, gender, and pack-years of smoking

\begin{tabular}{lccc}
\hline Co-morbidity score & HR & $(95 \% \mathrm{CI})$ & $\mathrm{P}$ \\
\hline ACE-27 Prognostic co-morbidity & 1.99 & $(1.17-3.39)$ & 0.011 \\
ACE-27 co-morbidity Grade (0-3) & 1.29 & $(1.01-1.67)$ & 0.043 \\
Charlson co-morbidity Index (0- $\geq 5)$ & 1.14 & $(.94-1.38)$ & 0.194 \\
\hline
\end{tabular}

had nearly twice the risk of death as those without. We suspect that the dichotomomized ACE-27 performs as well or better than the categorical ACE-27 score because there is no difference in prognosis among the lower comorbidity scores (0-2). This differs from previous study of the ACE-27 where each score (0-3) had a different prognosis [18].

Validating the ACE-27 as a co-morbidity instrument for use in COPD is an important advance for the study of this disease. The uses of such a tool are multifaceted, including assessing adequate randomization in clinical trials, risk adjustment in health services research, and adjusting for co-morbidity as a potential confounder in observational studies.

Our concomitant evaluation of the CCI had mixed results. It

did not detect a significant violation of the proportional hazards assumption in these models.

We reviewed the cases of the 20 patients with ACE-27 prognostic co-morbidity but low Charlson scores. We found that 8 of these cases had high ACE-27 scores because they collected information on co-morbidity not collected by the CCI. The most frequent examples were chronic arrhythmias, hypertension, and alcohol abuse. In another 8 cases both the ACE- 27 and CCI collected the presence of the comorbidities but the ACE-27 scored the burden of co-morbidity higher because it measured the severity of the co-morbidities. The most common examples were recent myocardial infarction and recent admission for congestive heart failure. In the remaining 4 cases, both ACE-27 and CCI collected information on the disease and disease severity.

\section{Discussion}

Co-morbidity measured by the ACE-27 was a significant predictor of survival in patients with COPD. Patients with "prognostic co-morbidity" was a univariate predictor of mortality with an estimated $18 \%$ increase in risk of death for every increase of one in the Charlson index. However this association disappeared in the multivariable analysis. Possible explanations for this include a loss of power in the multivariate analysis, confounding, or both. We suspect that confounding is responsible because there was a $>10 \%$ change in the hazard ratios between the univariate and multivariate analysis with very little change in the breadth of the confidence intervals. Therefore the CCI adds no useful information beyond that which age, gender, and years of smoking provide.

Our study was not designed to test whether the ACE-27 and the CCI were different in their predictive validity. More subjects and a comparative analysis would be required to test that hypothesis. Thus we cannot say that the apparent difference in performances are due to more than random chance. However we did compare the two co-morbidity scores to generate ideas for why the ACE-27 may have performed better. Our informal analysis suggests that the ACE-27 may have had an advantage by collecting more conditions and by grading the 
severity of conditions that both the CCI and ACE27 collected. It collected important conditions such as arrhythmias, hypertension, and alcohol abuse that have been shown in prior studies to affect survival [29-31]. Furthermore, the ACE-27 appears to have an advantage by grading the severity of heart failure and myocardial infarction which also has been previously shown to predict survival $[17,32,33]$.

This study has certain limitations. This was an American, Caucasian population in a rural state and the results may not be generalizable to other populations where the relationship between comorbidity and mortality may differ. The chart reviewer was not blinded to death during the admission while collecting information on co-morbid conditions. The low number of deaths during the admission make this potential measurement bias unlikely to affect the results.

We have demonstrated that the ACE-27 is a valid measure for use when considering the relationship between co-morbidity and survival. Further research is needed to confirm whether the ACE-27 is truly superior to the Charlson Co-morbidity Index for predicting survival in patients with COPD.

\section{References}

1. Burrows B, Earle RH. Course and prognosis of chronic obstructive lung disease: A prospective study of $200 \mathrm{pa}-$ tients. NEJM 1969; 280: 397-404.

2. Hansell AL, Walk JA, Soriano JB. What do chronic obstructive pulmonary disease patients die from? A multiple cause coding analysis. Eur Respir J 2003; 22: 809814.

3. Mannino DM, Brown C, Giovino GA. Obstructive lung disease deaths in the United States from1979 through 1993. An analysis using multiple-cause mortality data. Am J Respir Crit Care Med 1997; 156: 814-818.

4. Wijnhiven HA, Kriegsman DM, Hesselink AE, De Hann M, Schellevis FG. The influence of co-morbidity on health -related quality of life in asthma and COPD patients. Respir Med 2003; 97: 468-475.

5. Van Manen JG, Bindels PJ, Ijzermans CJ, Van Der Zee JS, Bottema BJ, Schade E. Prevalence of co-morbidity in patients with a chronic airway obstruction and controls over the age of 40. J Clin Epid 2001; 54: 287-293.

6. Patil SP, Krishnan JA, Lechtzin N, Diette GB. In-hospital mortality following acute exacerbation of chronic obstructive pulmonary disease. Arch Intern Med 2003; 163: 1180-1186.

7. Fan VS, Curtis JR, Tu SP, McDonell MB, Fihn SD. Using quality of life to predict hospitalizations and mortality in patients with obstructive lung disease. Chest 2002; 122: 429-436

8. Soriano JB, Vestbo J, Pride NB, Kiri V, Maier WC. Survival in COPD patients after regular use of fluticisone proprionate and salmeterol in general practice. Eur Respir J 2002; 20: 819-825.

9. Almagro P. Calbo E. Ochoa de Echaguen A., et al. Mortality after hospitalization for COPD. Chest 2002; 121: $1441-8$

10. Yohannes AB, Baldwin RC, Connolly M. Mortality predictors in disabling chronic obstructive pulmonary disease in old age. Age and Ageing 2002; 31: 137-140.

11. Domingo-Salvany A, Lamarca R, Ferrer $\mathrm{M}$, et al. Health-related quality of life and mortality in male patients with chronic obstructive pulmonary disease. Am J Respir Crit Care Med 2002; 166: 680-685.

12. Incalzi RA, Fuso L, De Rosa M, et al. Co-morbidity contributes to predict mortality of patients with chronic obstructive pulmonary disease. Eur Respir J 1997; 10: 2794-2800.

13. Groenwgen KH, Schols AM, Wouters EF. Mortality and mortality-related factors after hospitalization for acute exacerbations of COPD. Chest 2003; 124: 459467.

14. Charlson ME, Pompei P, Ales KL, MacKenzie RC. A new method of classifying prognostic co-morbidity in longitudinal studies: development and validation. $J$ Chronic Dis 40: 373-383.

15. Iezzoni LI. Dimensions of risk. In Iezzoni LI ed; Risk adjustment for measuring healthcare outcomes. $2^{\text {nd }}$ ed. Chicago, Ill: Health Administration Press, 1997: 73-78.

16. Hulley SB, Martin JN, Cummings SR. Planning the measurements: precision and accuracy. In: Hulley SB, Cummings SR, Browner WS, Grady D, Hearst N, Newman TB eds. Designing Clinical Research 2nd ed. Philadelphia, PA: Lippincott Williams \& Wilkins, 2001: 44.

17. Loh E. Prognosis of heart failure. In: UpToDate v10.2 Rose BD ed. Wellesley, MA: UpToDate, 2002.

18. Piccirillo, JF, Costas I, Claybour P, Borah AJ, Grove L. The measurement of co-morbidity by cancer registries. J Registry Management 2003; 30: 8-14.

19. Piccirillo JF. The impact of co-morbidity in head and neck cancer. J Registry Management 2001; 28: 173175.

20. Standards for the diagnosis and care of patients with chronic obstructive pulmonary disease (COPD) and asthma. American Rev Respir Dis 1997; 136: 225-44.

21. Burrows B, Bloom JW, Gayle AT, Cline MG. The course and prognosis of different forms of chronic airways obstruction in a sample from the general population. NEJM 1987; 317: 1309-1314.

22. Burrows B. The course and prognosis of different types of chronic airflow limitation in a general population sample from Arizona: Comparison with the Chicago “COPD” series. Am Rev Respir Dis 1989; 140: S92-s94.

23. Meyer PA, Mannino DM, Redd SC, Olson DR. Characteristics of adults dying with COPD. Chest 2002; 122: 2003-2008.

24. Goel A, Pinckney RG, Littenberg B. APACHE II predicts long-term survival in COPD patients admitted to a general medical ward. J Gen Intern Med 2003; 18: 824-30.

25. Ancestry. com [Internet] Provo (Utah): MyFamily. com, Inc. Available from: http: //www. ancestry. com/search/ rectype/vital/ssdi/main. htm

26. Kaplan MH, Feinstein AR. The importance of classifying initial co-morbidity in evaluating the outcome of diabetes mellitus. J Chronic Dis 27: 387-404.

27. Grambsch PM, Therneau TM. Proportional hazards tests and diagnostics based on weighted residuals. Biometrica 1994; 81: 515-526.

28. StataCorp. 1999. Stata Statistical Software: Release 6. 0. College Station, TX: Stata Corporation.

29. ACC/AHA/ESC Guidelines for the management of patients with atrial fibrillation: executive summary. $J \mathrm{Am}$ Coll Cardiol 2001; 38: 1231-65.

30. Sixth report of the joint national committee on prevention, detection, evaluation, and treatment of high blood pressure. Arch Intern Med 1997; 157: 2413-2446.

31. Gold MS, Aronson MD. Screening and diagnosis of patients with alcohol problems. In: UpToDate v10.2 Rose BD ed. Wellesley MA: UpToDate, 2002.

32. Alpert J. Risk stratification for cardiac events after acute myocardial infarction. In: UpToDate v10.2 Rose BD ed. Wellesley MA: UpToDate, 2002.

33. Bronson DL, Halperin AK, Marwick TH. Evaluating cardiac risk in noncardiac surgery patients. Cleve Clin J Med 1995; 62: 391-400. 